\title{
CrystEngComm
}

Check for updates

Cite this: CrystEngComm, 2020, 22, 7380

Received 16th March 2020

Accepted 6th May 2020

DOI: $10.1039 / \mathrm{d} 0 \mathrm{ce} 00403 \mathrm{k}$

rsc.li/crystengcomm

\section{Chirality and stereoisomerism of organic multicomponent crystals in the CSD $\uparrow$}

\author{
Eline Grothe, (iD) Hugo Meekes (iD) and René de Gelder (iD*
}

\begin{abstract}
With the current interest in multicomponent crystals containing chiral residues, a wide variety of studies could benefit from a comprehensive inventory of chirality in multicomponent crystals. We combined computational approaches for identifying chiral carbon atoms and methods for identifying residue types in order to make such an inventory for all organic multicomponent entries in the Cambridge Structural Database (CSD). [Groom et al., Acta Cryst. B, 2016, 72, 171-179] This inventory provides a new and extended view on multicomponent classification by including chirality. We classified 66355 multicomponent CSDentries into one of seven multicomponent classes and one of seven stereoisomerism classes, based on the residue type and the presence of chirality in each entry. We present refcode lists of the 49 resulting subclasses, and examples of applications of the combined classification.
\end{abstract}

\section{Introduction}

A variety of studies report the influence of chirality and the type of multicomponent system on properties like crystallographic symmetry and enantioselectivity. ${ }^{1-5}$ For example, the crystallization behaviour of racemic mixtures in multicomponent systems has been shown to vary greatly between different types of multicomponent systems, as well as between multicomponent and unary systems. Springuel et al. compared the cocrystallization behaviour of racemic mixtures combined with enantiopure coformers to that of diastereomeric salts. ${ }^{6}$ Crystallization of salts is known to result in diastereomeric salt formation, where the enantiopure counterion crystallizes with both enantiomers at different solubilities. Springuel et al. showed that 38 out of 44 of these cocrystals found in the Cambridge Structural Database (CSD),${ }^{6,7}$ show enantiospecificity, meaning that the enantiopure coformer only cocrystallizes with one of the enantiomers in the racemic mixture. A subsequent experimental screening on new systems affirmed this finding: 13 out of 15 cocrystal systems showed enantiospecificity. ${ }^{6}$

In another study, Cruz Cabeza et al. investigated 3921 binary solvates of chiral and achiral molecules in a data mining study in 2007, and revealed that the space group

Institute for Molecules and Materials, Radboud University, Nijmegen, The Netherlands. E-mail: r.degelder@science.ru.nl

$\dagger$ Electronic supplementary information (ESI) available: 31 CSD-refcode lists of subsets resulting from the classification; one CSD-refcode list of 90 multicomponent kryptoracemates; one CSD-refcode list of 165 cocrystals containing diastereomeric pairs; a list of solvents known to the algorithm used; a summary of rejected entries. All are supplied as txt files. See DOI: 10.1039/ doce00403k occurrence of solvates greatly varies with the solvent. ${ }^{4}$ Space group choice is an important issue in crystal structure prediction (CSP), where crystal structures are usually generated for only a selection of space groups. Often, about ten of the most common space groups are used, ${ }^{8-10}$ which corresponds to $88 \%$ of the crystal structures in the CSD. ${ }^{11}$ By determining the space group distribution in solvates, Cruz Cabeza et al. showed that CSP of some solvates can be performed on less than ten space groups without reducing the confidence of including the correct space group.

As part of a database study into the stability of binary cocrystals Gavezzotti et al. found that the frequency of Sohncke space groups in binary cocrystals is much lower than in unary crystals. ${ }^{12}$ As Pidcock determined that over $50 \%$ of chiral compounds found in the CSD crystallize in a Sohncke space group, in contrast to $10 \%$ of achiral entries, ${ }^{5}$ Gavezzotti et al. hypothesized that there may be a low fraction of chiral molecules in the binary cocrystal set. However, they had no means to directly determine the fraction of chiral molecules in their set. With an inventory of all chiral molecules in binary cocrystals we would be able to verify or falsify their hypothesis.

About half of all active pharmaceutical ingredients (APIs) are chiral compounds, most of which have been marketed as racemic compounds, such as ibuprofen (a nonsteroidal antiinflammatory drug), or propranolol (a $\beta$-blocker). ${ }^{13}$ Owing to technological advances in chiral separation and asymmetric syntheses, the US Food and Drug Administration (FDA) officially promotes the development of new chiral drugs as enantiopure compounds since $1992 .{ }^{14}$ The separation of enantiomers from a racemic mixture (chiral resolution) can be achieved by a variety of methods, such as chiral 
chromatography, diastereomeric salt formation, and conglomerate crystallization. $^{15-17}$ Enantiospecific cocrystallization $^{3}$ and conglomerate cocrystallization ${ }^{18}$ have been reported as possible alternatives for chiral resolution of racemic compounds that do not easily form salts or racemic conglomerates. These enantiopure cocrystals of APIs are also attractive from a crystal-engineering point of view, because multicomponent crystals can be designed to improve physicochemical properties of the deliverable without modifying the API. ${ }^{19}$ This again underlines the importance of understanding the influence and occurrence of chirality in multicomponent crystals.

In this contribution, we will make a comprehensive inventory of chirality in multicomponent crystals through data mining. We identify carbon stereocenters and determine the relative configuration of residues to distinguish opposite enantiomers and configurational diastereomers; other types of chirality are excluded (e.g. $P$ or $S$ stereocenters, and axial chirality). Our combined stereoisomeric and multicomponent classification leads to 49 subclasses that are provided as lists of CSD refcodes. We will show how the results can serve various goals: e.g. the study of enantiospecific behaviour of cocrystals and space group choice in CSP. Additionally, we will determine both the fraction of chiral molecules in binary cocrystals in the CSD and in Gavezzotti's cocrystal dataset, to verify the hypothesis on the lack of Sohncke space groups in this set.

\section{Nomenclature}

\subsection{Multicomponent classification}

A residue is considered to be a complete set of covalently bonded elements. In this contribution, covalent bonds will be defined by the connectivity records of the datafile used. Two residues are considered different when the connectivity, elements or relative configuration is different. The latter qualification occurs when two residues with otherwise identical connectivity have chiral centres whose configuration is non-identical, non-opposite. This means that for the multicomponent classification configurational diastereomers are considered different residues, but enantiomers are not. In our search for diastereomers we have only focussed on cases that have one or more different stereocenters; we did not include geometric ( $E / Z$ or cis/trans) isomers.

Now we can define multicomponent crystals as crystals with two or more different residues. We refer to the number of different residues in the crystal as $Z^{\mathrm{R}}$ such that $Z^{\mathrm{R}}>1$ for multicomponent crystals and $Z^{\mathrm{R}}=1$ for single-component (or 'unary') crystals.

We adhere to the classification system as defined by Grothe et al. in $2016,{ }^{20}$ by defining three types of residues ions, solvents, and coformers - as follows:

Ion residue with a nonzero formal charge.

Solvent neutral residue, liquid at ambient conditions.

Coformer neutral residue that is not a solvent.
We use the atomic charges in the data file to determine the net charge of each residue and to identify ions. Zwitterions have no net charge and are not considered ions. We use a list of 100 known solvents to identify solvents and thereby solvates. This list includes the most common solvents used for the crystallization of organic and organometallic compounds. ${ }^{21}$

With this definition of residue and residue type we can distinguish seven different types of multicomponent crystals, outlined in Table 1 and Fig. 1(a). ${ }^{20}$ Crystals comprised of only solvents are not included in this classification.

\subsection{Stereoisomeric classification}

The chirality of $\mathrm{sp}^{3}$ carbon atoms is determined by the covalent connectivity and atomic number of its four substituents. ${ }^{22}$ If none of the substituents are identical the carbon is chiral.

In terms of chirality we can distinguish between chiral and achiral residues; the latter can be mesoisomeric or nonchiral:

Chiral non-superposable with its mirror image.

Achiral superposable with its mirror image.

Nonchiral achiral residue without chiral centres.

Mesoisomer achiral residue with chiral centres.

Our algorithms identify chiral residues with chiral carbons. If for each chiral centre an equivalent centre of opposite chirality is found, then the structure will be identified as a mesoisomer.

With this definition of mesoisomers, chiral and nonchiral residues, we can distinguish seven different types of stereoisomeric crystals, as follows:

True nonchiral only nonchiral residues.

True chiral only chiral residues.

True meso only meso residues.

Chiral nonchiral both chiral and nonchiral residues.

Meso nonchiral both meso and nonchiral residues.

Meso chiral both meso and chiral residues.

Meso chiral nonchiral meso, chiral and nonchiral residues.

This is summarized in Table 2 and visualized in Fig. 1(b).

\subsection{Stereoisomeric multicomponent classification}

Table 2 presents seven mutually exclusive classes of stereoisomerism in crystals. When we combine the

Table 1 Number of each residue type for the subclasses that are used here. The class of unary crystals is listed for completeness

\begin{tabular}{lrcc}
\hline & Ion & Solvent & Coformer \\
\hline Unary & 0 & 0 & 1 \\
True solvate & 0 & $\geq 1$ & 1 \\
True salt & $\geq 2$ & 0 & 0 \\
True cocrystal & 0 & 0 & $\geq 2$ \\
Salt solvate & $\geq 2$ & $\geq 1$ & 0 \\
Cocrystal solvate & 0 & $\geq 1$ & $\geq 2$ \\
Cocrystal salt & $\geq 2$ & 0 & $\geq 1$ \\
Cocrystal salt solvate & $\geq 2$ & $\geq 1$ & $\geq 1$
\end{tabular}




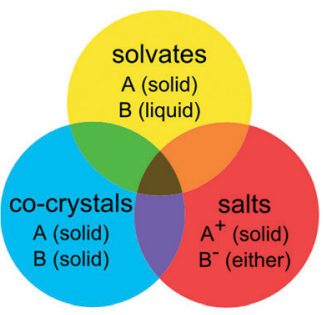

(a)

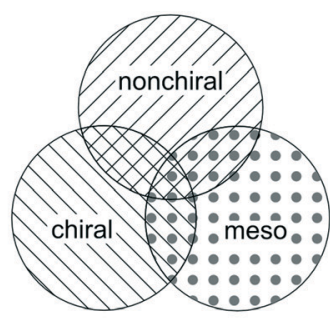

(b)

Fig. 1 Three overlapping classes (a) multi-component classes* and (b) stereoisomeric classes, visualized as circles. Each color represents one multicomponent class from Table 1, each filling represents one stereoisomeric class from Table 2. *Reprint of multicomponent classification as presented in ref. 20.

stereoisomeric with the multicomponent classification we can distinguish 49 subclasses of multicomponent crystals based on their stereoisomerism and residue types, as in Fig. 2.

As examples of this classification, Fig. 3 shows three different crystal structures found in the CSD. CSD-entry ZULCIU ${ }^{23}$ in Fig. 3(a) is a true meso cocrystal: it contains two coformers both of which are mesoisomers. Interestingly, the two mesoisomers differ from one another only by relative configuration and are therefore diastereomers. Remember that in terms of classifying multicomponent crystals, diastereomers are considered different residues.

CSD-entry YASGEG ${ }^{24}$ in Fig. 3(b) is a true chiral cocrystal: it contains two coformers, both of which are chiral. This crystal of levetiracetam ((S)-2-(2-oxopyrrolidin-1-yl)butanamide) and D-tartaric acid was obtained by Springuel et al. ${ }^{24}$ and is an example of enantiospecific cocrystallization, meaning that the levetiracetam L-tartaric acid cocrystal does not form. ${ }^{3}$

CSD-entry MAXBET ${ }^{25}$ in Fig. 3(c) is a meso chiral nonchiral cocrystal solvate: it contains two coformers and one solvent; one of the coformers is chiral, the other is a mesoisomer, the solvent - benzene - is a nonchiral residue.

\subsection{List of terms}

Besides the classification terms defined above, we also use several terms in our analysis, that might be worth defining in advance:

Sohncke space group space group containing no mirror nor inversion symmetry operations.

Racemate equimolar mixture of a pair of enantiomers (in any state).

Table 2 Number of each stereoisomeric type for the subclasses that are used here

\begin{tabular}{lrrr}
\hline & Nonchiral & Meso & Chiral \\
\hline True nonchiral & $\geq 1$ & 0 & 0 \\
True meso & 0 & $\geq 1$ & 0 \\
True chiral & 0 & 0 & $\geq 1$ \\
Meso nonchiral & $\geq 1$ & $\geq 1$ & 0 \\
Chiral nonchiral & $\geq 1$ & 0 & $\geq 1$ \\
Meso chiral & 0 & $\geq 1$ & $\geq 1$ \\
Meso chiral nonchiral & $\geq 1$ & $\geq 1$ & $\geq 1$
\end{tabular}

Conglomerate crystal enantiopure crystal formed from a racemate.

Conglomerate cocrystal enantiopure cocrystal formed from a racemic mixture of enantiomers and a coformer.

\section{Methods}

ConQuest 2.03 was used to search the CSD 5.40 database (including updates up to and including May 2019) for entries with the following flags: "3D-coordinates determined", "Not disordered", "No errors", "Not polymeric", "Organics". We exported the entries to files with pdb and mol2 format and for repeated refcodes discarded all but the highest sequential number, and passed these files to our in-house program Chichi. $^{26}$ The majority of the discarded refcodes are redeterminations, however, some polymorphs will inevitably also be discarded with this selection. ${ }^{27}$

The multicomponent classification is based on the types of residue in an entry: ion, solvent molecule, or coformer, and follows the rules outlined in Table 1. In earlier work, only ions and non-ions were identified automatically by Maruchi (a separate algorithm), and we would identify solvents separately by generating a hit list in ConQuest for each known solvent. $^{20}$ However, we now incorporated determination of residue types in the latest version of

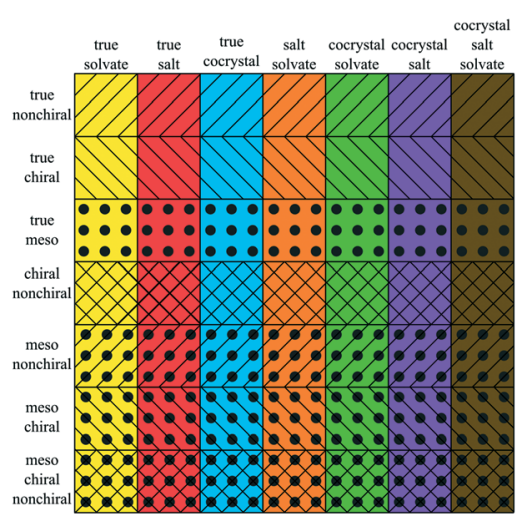

Fig. 2 Tabular visualization of how the two classification systems overlap to form 49 subclasses. Rows: Classes of stereoisomerism (shown as stripes and dots), columns: multicomponent classes (colored). 


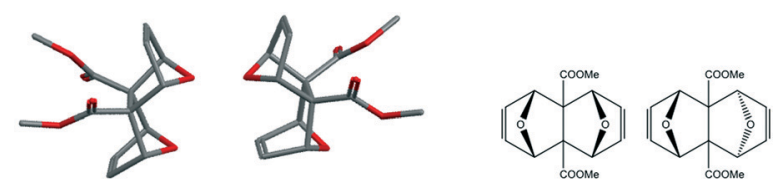

(a)

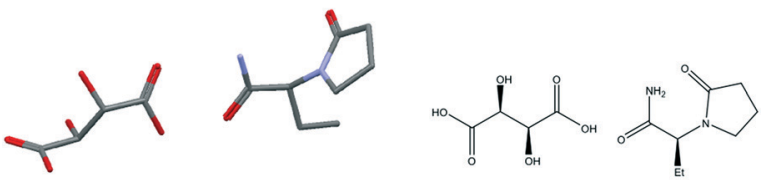

(b)
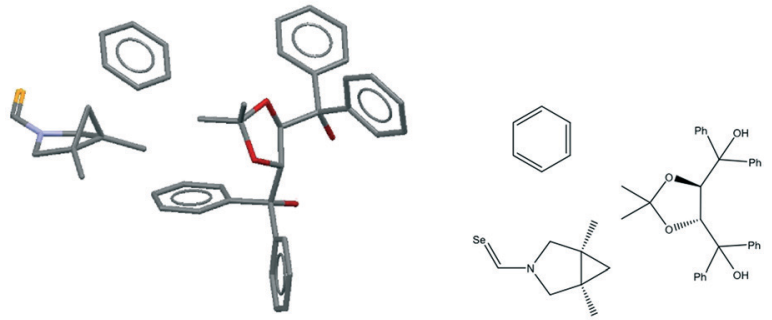

(c)

Fig. 3 The asymmetric units (left) and structural formulae (right) for three CSD-entries. (a) ZULCIU: ${ }^{23}$ a true meso cocrystal. Both coformers are mesoisomers and form a diastereomeric pair. (b) YASGEG: ${ }^{24}$ a true chiral cocrystal. Both coformers are chiral residues. (c) MAXBET: ${ }^{25}$ a meso chiral nonchiral cocrystal solvate. Both coformers have chiral centres, but only one is chiral; the solvent is nonchiral.

Chichi. Given an input file of atom and bond records of known solvents Chichi distinguishes solvents from coformers automatically. A list of 100 known solvents used in this research is available as ESI. $\dagger$ Water is identified by checking for neutral residues consisting of one $\mathrm{sp}^{3}$ oxygen atom and two hydrogen atoms. Some hydrates will not be classified as solvates in case the water molecule in the model is incomplete, e.g. FUPBUN. ${ }^{28}$

Chichi determines the stereoisomerism of entries by first finding chiral carbons in the entry's residues, and then comparing all the entry's chiral residues. Its methods have been described in detail previously. ${ }^{26}$ The algorithm is limited to carbon stereocenters; other types of chirality (e.g. $P$ or $S$ stereocenters and axial chirality) are not considered. A key step involves ranking the atoms by atom type and topology, which allows for sorting the atoms and thereby for the comparison of two residues or two substituents. Hydrogen atoms are not included in topology as determined by Chichi. For the ranking procedure, atom numbers are used as atom type descriptors and atomic walk count sequences are used as descriptors of atom topology. After sorting the atoms by their ranks, two residues in an entry or two substituents of a potentially chiral carbon can be compared by sorted atom types and sorted atomic walk count arrays. For chiral carbons, the sign of chirality is determined by determining the coordinates of the three heaviest ranked substituents. This algorithm distinguishes two enantiomers, but does not reproduce $R$-S-labels often used in nomenclature.

In addition to the algorithm described previously ${ }^{20}$ we let Chichi also read the "SYBYL atom types" from the atom records in the mol2 file. These types are used as a last step in ranking the atoms and as a last step in comparing residues before comparing their chirality. SYBYL types are also relevant for distinguishing between solvents (and small molecules in general) such as cyclohexane and benzene, which cannot be distinguished based on atom types and topology alone.

Some data inconsistencies are known to occur in the CSD. Entries with erroneous or incomplete records or inconsistent bond types might be misclassified by Chichi. To prevent misclassifications based on charge, entries with a net charge are flagged by Chichi.

To prevent misclassifications based on SYBYL types, ChiChi only uses the following SYBYL types: $\mathrm{sp}, \mathrm{sp}^{2}, \mathrm{sp}^{3}$, ar, $\mathrm{pl} 3, \mathrm{Du}$, and the remainder is classified as unknown. ChiChi converts N.4, N.am, S.o, and S.o2 to $\mathrm{sp}^{3}$, C.cat to $\mathrm{sp}^{2}$, and pairs of $\mathrm{O} . \mathrm{co} 2$ to $\mathrm{sp}^{2}$ and $\mathrm{sp}^{3}$. Additionally, nitrogen atoms in contrast to other atom types, are not ranked by SYBYL type, because the N.pl3 type is very inconsistently assigned and can refer to $\mathrm{sp}^{2}, \mathrm{sp}^{3}$, or aromatic nitrogen (DIMPEW, ${ }^{29}$ YUZXUO $^{30}$ ACINOP $^{31}$ respectively). When comparing two residues $\mathrm{pl} 3$ nitrogen is a wildcard for $\mathrm{pl} 3, \mathrm{sp}^{2}, \mathrm{sp}^{3}$ and ar nitrogen.

Chichi analyzed and classified 309588 entries after which we rejected 6932 entries. Most of the rejected entries had a
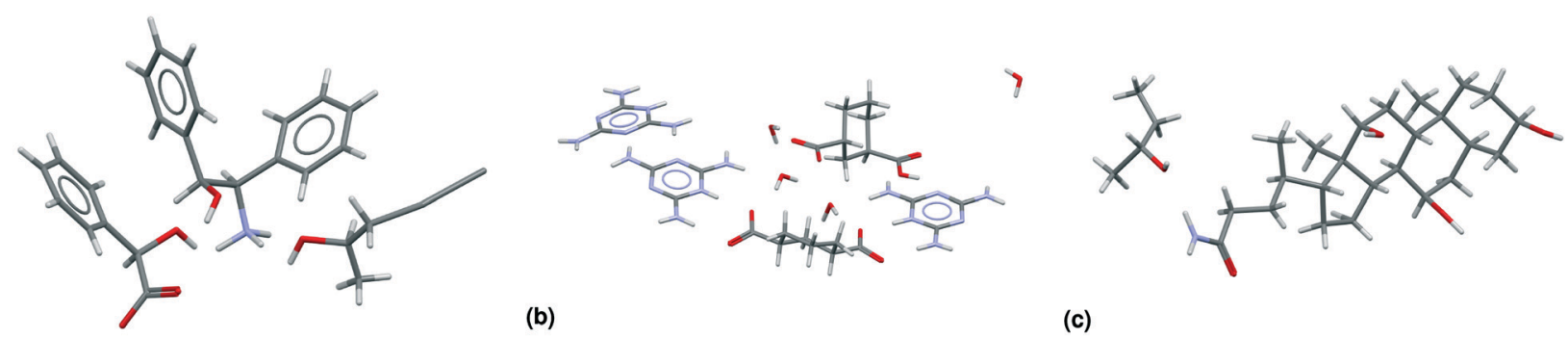

(b)

(c)

(a)

Fig. 4 Asymmetric units of CSD-entries in the MULTI set. (a) KOTNEO ${ }^{34}$ is the only entry in its subclass: a true chiral salt solvate. It was initially classified as a cocrystal salt, because the solvent (pentan-2-ol) is not included in our list of solvents. (b) JUMYIB ${ }^{35}$ is a meso chiral nonchiral salt solvate. (c) TEHPO ${ }^{36}$ is a true chiral true solvate. 
net charge in the asymmetric unit. A summary of entry rejections can be found in the ESI. $\dagger$ For 2 entries the space group was manually added, as the given space group is not recognized by ChiChi: " $B 2 / c$ " in ZZZIYE06 (ref. 32 ) and " $I 2 / m 11$ " in NIYBOM01 (ref. 33) (ChiChi recognizes all settings available in the ConQuest space group query). The results in the following section are based on the remaining 302656 entries.

\section{Results and discussion}

Table 3 shows the classification of the 66355 multicomponent entries in our dataset, a subset we will refer to in this paper as MULTI. Not all 49 subclasses (Fig. 2) are populated. For example, the only true meso crystals we found are true cocrystals; none of the other multicomponent classes contain a true meso entry. One of these true meso crystals, ZULCIU $^{23}$ is shown in Fig. 3(a). Some other subclasses are existent, but rare, such as meso chiral true salts (5 entries in MULTI), meso chiral nonchiral salt solvates (2 entries). One entry, KOTNEO ${ }^{34}$ was classified as a true chiral cocrystal salt, however, the supposed coformer was actually a solvent that is not included in our list of solvents known to the algorithm (pentan-2-ol). For clarity, the entry was manually reclassified in the $\mathrm{ESI}^{\dagger}$ and in Table 3 as a true chiral salt solvate, the only entry in its class. The crystal structure of KOTNEO is shown in Fig. 4(a).

There are only four cases of meso chiral nonchiral crystals in the MULTI set of 66355 entries. Fig. 3(c) already showed an example of a meso chiral nonchiral cocrystal solvate; Fig. 4(b) shows another crystal structure, this one a salt solvate, JUMYIB. ${ }^{35}$ None of the other multicomponent classes contain entries that have meso, chiral, as well as nonchiral residues. Fig. 4(c) shows a true chiral true solvate, TEHPOL; ${ }^{36}$ only 13 true solvates out of 24654 are true chiral.

In the ESI $\dagger$ refcode lists for all of the 31 populated subclasses can be found. In the following sections, we will discuss application of this classification system to studies of space group frequency, enantiospecificity and kryptoracemates. In earlier work, we published a list of kryptoracemates, which is an interesting subset of enantiomers that crystallize in a Sohncke space group. ${ }^{26}$ In section 4.3 this subset will be discussed once more, and a refcode list of multicomponent kryptoracemates is supplied in the ESI† as well.

\subsection{Multicomponent stereoisomeric classification}

Stereoisomerism and space group frequency. An important aspect in crystal structure prediction (CSP) is the selection of the correct space group when generating crystal structures. Owing to computational costs, it is often not feasible to generate crystal structures for all 230 space groups to be assured to include the correct space group. Instead, space groups in CSP are usually limited to the ten most frequent ones. It is therefore important to know which space groups are most frequent for the stereoisomeric and multicomponent subset the system of interest belongs to. For solvates, Cruz Cabeza et al. ${ }^{4}$ showed a significant difference in space group frequencies between chiral coformers (which they refer to as ${ }^{\mathrm{C}} M$ ) and achiral coformers $\left({ }^{\mathrm{A}} M\right)$. Looking at our MULTI set, we expect to see similar results when we compare the true nonchiral true solvates with the chiral nonchiral true solvates. Fig. 5(a) shows the space group frequencies for these two MULTI subsets as well as the results for ${ }^{\mathrm{A}} M$ and ${ }^{\mathrm{C}} M$; only the four most common Sohncke and four most common non-Sohncke space groups are shown. Even though the data sets are somewhat different (MULTI includes all true solvates, ${ }^{\mathrm{C}} M$ and ${ }^{\mathrm{A}} M$ only binary solvates excluding hydrates; ${ }^{\mathrm{C}} M$ includes mesoisomers, the chiral nonchiral set does not), the distributions are very similar. The non-Sohncke space groups constitute the vast majority of true nonchiral $/^{\mathrm{A}} M$ solvates; the solvates including a chiral molecule crystallize most frequently in $P 2_{1} 2_{1} 2_{1}$ and $P 2_{1}$.

The trend was initially observed by Cruz-Cabeza et al. in solvates; when we plot a similar graph for the whole MULTI set, we can see that this trend is in fact present for multicomponent crystals in general, see Fig. 5(b). The trend continues for true chiral entries (Fig. 5(b)), with $P 2_{1}$ becoming the most prevalent space group and $94.5 \%$ of the crystals occupying a Sohncke space group.

The NOCOR set. Gavezzotti et al. ${ }^{12}$ investigated a dataset of binary cocrystal entries in the CSD (the NOCOR set) and found the Sohncke space group frequency being significantly reduced with respect to unary crystals. Based on these frequencies they proposed that chiral compounds might be underrepresented in the NOCOR set. Using our classification system we can determine the validity of the hypothesis that chiral compounds may be underrepresented in the true cocrystal class. To find all entries with any chiral compounds,

Table 3 The number of CSD entries for the stereoisomeric classes within each multicomponent class

\begin{tabular}{|c|c|c|c|c|c|c|c|c|}
\hline & True solvate & True salt & True cocrystal & Salt solvate & Cocrystal solvate & Cocrystal salt & Cocrystal salt solvate & Subtotal \\
\hline True chiral & 13 & 584 & 444 & 1 & 0 & 0 & 0 & 1042 \\
\hline True meso & 0 & 0 & 8 & 0 & 0 & 0 & 0 & 8 \\
\hline Chiral nonchiral & 8919 & 4549 & 903 & 1714 & 183 & 213 & 82 & 16563 \\
\hline Meso nonchiral & 599 & 209 & 161 & 75 & 26 & 36 & 9 & 1115 \\
\hline Meso chiral & 0 & 5 & 17 & 0 & 0 & 2 & 0 & 24 \\
\hline Meso chiral nonchiral & 0 & 0 & 0 & 2 & 2 & 0 & 0 & 4 \\
\hline
\end{tabular}




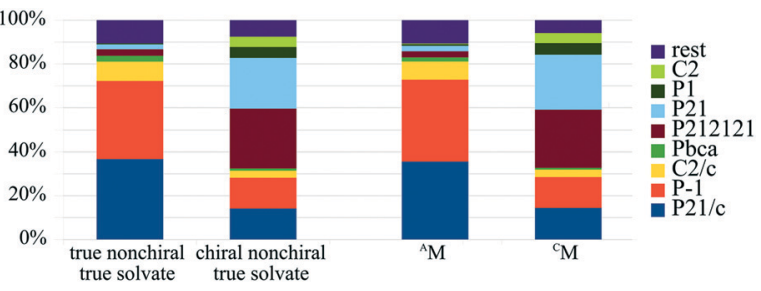

(a)

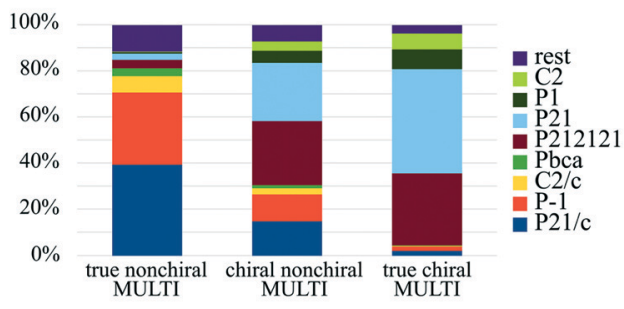

(b)

Fig. 5 Space group frequencies of the most common Sohncke and non-Sohncke space groups. (a) The two columns on the left are based on true nonchiral and chiral nonchiral true solvates in the MULTI set. Two columns on the right are based on the work by Cruz-Cabeza et al. on $Z^{R}=2$ solvates with coformers with $\left({ }^{C} M\right)$ and without $\left({ }^{A} M\right)$ a chiral centre. Note that ${ }^{C} M$ does not exclude mesoisomers. (b) Based on true nonchiral, chiral nonchiral and true chiral entries in the whole MULTI set.

we simply take the four stereoisomeric classes that contain chiral residues: true chiral, chiral nonchiral, meso chiral and meso chiral nonchiral. Of the 8338 true cocrystals in MULTI $16 \%$ of the entries contain chiral residues, whereas the entire MULTI set contains $27 \%$ entries with chiral residues, and for the unary entries this is as many as $42 \%$.

In the MULTI set the chiral compounds are indeed underrepresented in the true cocrystal class, but what about the NOCOR set? We can determine the representation of chiral compounds in the NOCOR set using the refcodes provided by Gavezzotti et al.: most of the NOCOR set is present in our MULTI set (1758 of 1941) of which Chichi identified 152 entries with a chiral residue (9\%). We can confirm Gavezzotti's hypothesis of underrepresentation of chiral compounds in the NOCOR set. Moreover, we can also add to the idea that this underrepresentation causes a

Table 4 Frequency of space group types in the NOCOR dataset compared to the complete true cocrystals set and unary crystals set, seperated into chiral and achiral entries

\begin{tabular}{lccc}
\hline & Centro & Non-centro & Sohncke \\
\hline Unary crystals & & & \\
Achiral & $85 \%$ & $7 \%$ & $9 \%$ \\
Chiral & $45 \%$ & $3 \%$ & $51 \%$ \\
True cocrystals & & & \\
Achiral & $90 \%$ & $4 \%$ & $5 \%$ \\
Chiral & $22 \%$ & $2 \%$ & $77 \%$ \\
True chiral & $8 \%$ & $2 \%$ & $91 \%$ \\
NOCOR & & & $5 \%$ \\
Achiral & $90 \%$ & $5 \%$ & $69 \%$ \\
Chiral & $31 \%$ & $0 \%$ & $100 \%$ \\
True chiral & $0 \%$ & $0 \%$ &
\end{tabular}

reduced frequency of Sohncke space groups by looking at the space group types occupied by chiral versus achiral entries in each of the compared subsets. This is done in Table 4 for the NOCOR subset, as well as the true cocrystals and unary crystals in our dataset, where each set is separated into crystals with and without chiral residues. We can see that the space group type frequency is strongly dependent on the chirality of the residues in the set: in the achiral sets the vast majority is centrosymmetric, whereas the chiral sets are mostly Sohncke. While $42 \%$ of all unary crystals contain a chiral residue, only $16 \%$ of true cocrystals, and $9 \%$ of the NOCOR subset does. Therefore, we can confirm Gavezzotti's suspicion that Sohncke space groups are less frequent in cocrystals due to a lower fraction of chiral compounds.

Interestingly, the frequency of Sohncke crystals of chiral residues is much higher in true cocrystals than it is in unary crystals. This may be at least partially explained by the type of studies that yield multicomponent crystals. More often than unary crystals, cocrystals are designed with a specific purpose, which in the case of chiral residues could be chiral resolution. The high frequency of true chirality in true cocrystals $(5 \%)$ is especially interesting given the underrepresentation of chiral residues in this set. This overrepresentation of true chiral entries in cocrystals could be caused by scientific interest in enantioselective or diastereomeric behaviour of cocrystals, because these systems contain multiple chiral residues.

True chiral multicomponent crystals contain only chiral residues. In Table 3 we can see that true chirality is less frequent in true solvates $(<1 \%)$ than in true salts and true cocrystals. This can be explained because true chiral true solvates must contain a chiral solvent, and most solvents are achiral.t

\subsection{Enantiospecificity and conglomerates}

One way to obtain enantiopurity is through spontaneous resolution, i.e. conglomerate crystallization. In case a chiral compound of interest does not form unary conglomerate crystals, multicomponent crystallization could provide an alternative route to spontaneous resolution. The numerous possible counterions, coformers and solvents are encouraging for the odds of finding a conglomerate system, but at the same time it is not feasible to test all systems experimentally. This is where the CSD can be of help. The enantiopure multicomponent entries containing the racemic compound of interest, could indicate as candidate systems, or one can look at the type of counterions, coformers and solvents that tend to crystallize with the racemic compound.

Only Sohncke space groups, having no inversion or mirror symmetry operations, allow for enantiopure crystals. To get an idea of how common enantiopurity is in multicomponent crystals, we can look at Fig. 6. The Sohncke frequency directly translates into frequency of enantiopurity, because the

$\ddagger$ Chiral solvents in our list include butane-2-ol, and 2-methylpiperidine. 


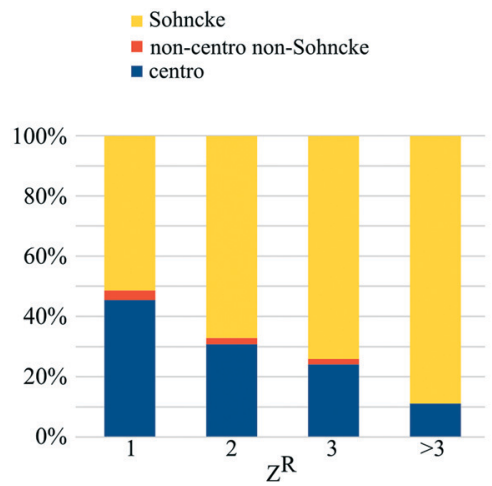

(a)

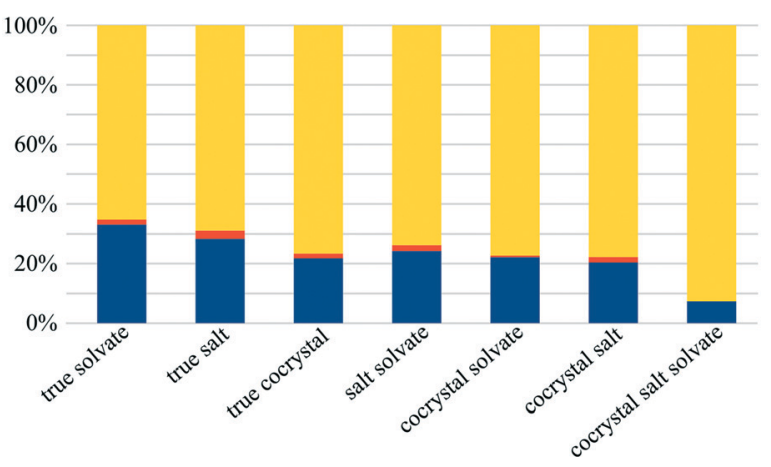

(b)

Fig. 6 Population of space group types: centrosymmetric (blue), Sohncke (yellow), and non-centrosymmetric and non-Sohncke (orange) for all entries that contain chiral residues, i.e. the stereoisomeric classes: true chiral, chiral nonchiral, meso chiral, meso chiral nonchiral. (a) Sorted by unary, binary, ternary and higher order crystals. (b) Sorted by type of multicomponent crystal.

number of kryptoracemates is negligible $(\leq 1 \%$ for all subsets). The frequency of enantiopure crystals increases with the number of residues: from $51 \%$ of unary crystals, to $88 \%$ of quaternary and higher order crystals.

Even though enantiopure crystals are more common for multicomponent systems, we cannot draw any conclusions on the frequency of conglomerates, because there is no way of telling whether an enantiopure entry is a conglomerate or was crystallized starting from enantiopure material, other than consulting the corresponding literature.

Another way to resolve a racemate is using an enantiospecific coformer. This is different from diastereomeric and from conglomerate crystallization in that only one of the enantiomers forms a crystal with the resolving agent. In contrast, in diastereomeric and conglomerate systems both enantiomers form crystals with the resolving agent, where in the case of diastereomeric systems, the crystals have different physical properties such as solubility. Springuel et al., who studied the differences between true chiral binary cocrystals and true chiral binary salts, found that while the salts usually form diastereomeric pairs of crystals, the cocrystal systems are often enantiospecific. ${ }^{6}$ To estimate the frequency of enantiospecificity, they consulted the literature for 52 cocrystals with two or more chiral residues in order to determine whether the systems are enantiospecific or "diastereomeric". The results of their literature study indicate that as much as $86 \% \pm 13 \%$ of cocrystals with multiple chiral residues are enantiospecific, and the rest are diastereomeric systems. This estimate can be improved by studying more crystals. The subclass of true chiral true cocrystals would be very suitable for this end: because true chiral entries consist of only chiral residues, these entries consist of multiple chiral residues similar to the 52 studied by Springuel $e t$ al. With our classification we have identified 444 true chiral true cocrystal entries in the MULTI set, all of which are binary cocrystals $\left(Z^{\mathrm{R}}=2\right)$. This set could be used in a literature study to improve the certainty of the enantiospecificity estimate calculated by Springuel et al., as this high percentage is promising for the use of resolution methods.

\subsection{Curiosities}

Multicomponent kryptoracemates. Most, but not all Sohncke crystals are enantiopure: a small portion of less than $1 \%$ contains an enantiomeric pair in the asymmetric unit. ${ }^{37}$ These so called kryptoracemates are relatively obscure systems, and are interesting for studying high $Z^{\prime}$-structures. We identified 90 multicomponent kryptoracemates, mostly salts and solvates, all of which can be found in the ESI. $\dagger$

In Table 5, the space group frequencies of all Sohncke crystals are compared to those of unary and multicomponent kryptoracemates. Mind, the size of the latter is only 90 entries. $P 2_{1} 2_{1} 2_{1}$ is the most populated Sohncke space group, and $P 2_{1}$ as the second most populated Sohncke space group. The frequency of $P 2_{1} 2_{1} 2_{1}$ drops from $49 \%$ for all Sohncke crystals to $26 \%$ for unary kryptoracemates and $16 \%$ for multicomponent kryptoracemates, while the frequencies of $P 2_{1}$ and $P 1$ space groups increase.

We would like to highlight CSD-entry YUBTEW ${ }^{38}$ in Fig. 7, perhaps the most exotic multicomponent kryptoracemate in the CSD: a chiral nonchiral kryptoracemic cocrystal salt solvate. Da Silva et al. ${ }^{38}$ obtained this crystal from a $1: 2$ molar ratio between lamivudine and racemic mandelic acid in a solvent mixture of water and ethyl alcohol. The crystal structure shows proton transfer only between the $R$-enantiomer of mandelic acid and lamivudine, forming a very robust ionic pair of lamivudine and $R$-mandelate, while the $S$-enantiomer acts as a neutral coformer. Three water molecules contribute to stabilizing the crystal structure.

Table 5 Most popular space groups for all Sohncke crystals, and for the subsets of unary and multicomponent kryptoracemates (unary k.rac. and multi k.rac)

\begin{tabular}{lrllr}
\hline & Entries & $P 2{ }_{1} 2_{1} 2_{1}$ & $P 2_{1}$ & \multicolumn{1}{c}{$P 1$} \\
\hline Sohncke & 79202 & $49 \%$ & $35 \%$ & $5 \%$ \\
Unary k.rac. & 462 & $26 \%$ & $50 \%$ & $18 \%$ \\
Multi k.rac. & 90 & $16 \%$ & $60 \%$ & $16 \%$
\end{tabular}




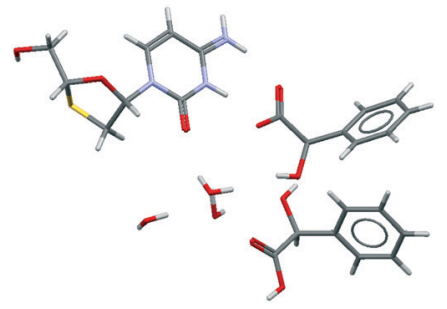

Fig. 7 The asymmetric unit of CSD-entry YUBTEW ${ }^{38}$ shows an ionic pair of lamivudine and $R$-mandelate, a $S$-mandelic acid coformer, and three water molecules. Lamivudine and the mandelic acid residues are chiral residues. The space group is $C 2$.

Table 6 The most popular space groups for all true chiral multicomponent crystals (true chiral multi) and the subset of true chiral crystals of configurational diastereomers (true chiral dias)

\begin{tabular}{lcccrr}
\hline & Entries & $P 2_{1}$ & $P 2_{1} 2_{1} 2_{1}$ & \multicolumn{1}{c}{$P 1$} & Other \\
\hline True chiral multi & 1042 & $45 \%$ & $31 \%$ & $9 \%$ & $15 \%$ \\
True chiral dias. & 130 & $48 \%$ & $8 \%$ & $23 \%$ & $22 \%$
\end{tabular}

Diastereomeric pairs. We also identified entries containing two or more configurational diastereomers, all of which can be found in the ESI. $\dagger$ This list of diastereomeric entries is in principle an extension of the list generated by Kelley et al. ${ }^{39}$ but we found that our method may lead to different interpretations of the same crystal structures. An explanation for these inconsistencies is that Kelley et al. use InChI strings to identify chirality while we use atomic walk count sequences as descriptors for the topology in molecules. Most of the 165 crystals of diastereomers we identified are true chiral (130), but in some entries one of the diastereomers is a mesoisomer, so they are classified as chiral meso entries. Interestingly, two crystals of diastereomers were found where both diastereomers are mesoisomers; one of these entries is shown in Fig. 3a, the other is CSD-entry EQOFIZ. ${ }^{40}$ All of the 130 true chiral crystals of diastereomers are true cocrystals. This means that $29 \%$ of all 444 true chiral true cocrystals contain two diastereomers.

In Table 6 the space group frequencies of true chiral diastereomers are compared to those of all true chiral multicomponent crystals. $P 2_{1}$ is the most populated space group for the complete set of true chiral multicomponent crystals $(45 \%)$ as well as being the most populated space group for the subset of true chiral diastereomeric crystals (48\%). $P 2_{1} 2_{1} 2_{1}$ and $P 1$ are at position two or three. Space group $P 2_{1} 2_{1} 2_{1}$ ranks second for the complete true chiral multicomponent set with $31 \%$, however, only $8 \%$ of the diastereomeric subset occupies this space group. Reversely, $P 1$ is occupied by $9 \%$ of true chiral multicomponent crystals, which is increased to $23 \%$ for the diastereomeric subset. This emphasizes how the nature of the residues strongly affects space group frequency.

\section{Conclusions}

We combined computational approaches for identifying chiral carbons and residue types in order to make an inventory for all organic multicomponent entries in the CSD. 66355 multicomponent CSD-entries were classified into 49 subclasses; 31 of which are actually populated. We provided refcode lists for the 49 subclasses, as well as refcode lists of multicomponent kryptoracemates and diastereomeric crystals.

The classification shows some interesting features, such as the underrepresentation of some subclasses (e.g. true chiral true solvates) and complete absence of others (e.g. true chiral salt solvates). While some of this is explained by the rarity of certain residues (e.g. chiral solvents), we have not been able to explain all numbers yet.

$22 \%$ of true cocrystals is in one of the chiral subclasses (i.e. true chiral, chiral nonchiral, meso chiral and meso chiral nonchiral) which is below average. At the same time as much as $5 \%$ of true cocrystals is true chiral, which is far above average. Decomposing all subsets is beyond the scope of this contribution, however, we identified several curiosities, including multicomponent kryptoracemates and diastereomeric cocrystals. It turns out that these diastereomeric entries greatly contribute to the high number of true chiral true cocrystals: 130 out of 444 true chiral true cocrystals consist of diastereomeric pairs.

The extended multicomponent classification with a classification based on chirality provides a more detailed picture of multicomponent crystals, and discloses the interplay between chirality and type of multicomponent system. Following the results of Cruz Cabeza et al. regarding space group frequencies of data sets with and without chiral coformers, we could confirm that true nonchiral true solvates occupy different space groups than chiral nonchiral true solvates. We showed the same difference is found for all true nonchiral multicomponent systems versus all chiral nonchiral multicomponent systems. We were also able to verify the hypothesis of Gavezzotti et al. that chirality is underrepresented in true cocrystals.

This study illustrates the enormous diversity in crystal structures found in the CSD. Studies that draw statistics from the CSD can benefit from larger data sets which can easily be created using the here presented classification and refcode lists.

\section{Conflicts of interest}

There are no conflicts to declare.

\section{Acknowledgements}

This research is supported by the Dutch Technology Foundation STW, which is part of the Netherlands Organization for Scientific Research (NWO), and which is partly funded by the Ministry of Economic Affairs.

\section{Notes and references}

1 T. Rekis, Acta Crystallogr., Sect. B: Struct. Sci., Cryst. Eng. Mater., 2020, 76, DOI: 10.1107/S2052520620003601. 
2 I. J. Scowen, T. S. Alomar, T. Munshi and C. C. Seaton, CrystEngComm, 2020, DOI: 10.1039/D0CE00301H.

3 G. Springuel and T. Leyssens, Cryst. Growth Des., 2012, 12, 3374-3378.

4 A. J. Cruz Cabeza, E. Pidcock, G. M. Day, W. D. S. Motherwell and W. Jones, CrystEngComm, 2007, 9, 556-560.

5 E. Pidcock, Chem. Commun., 2005, 3457-3459.

6 G. Springuel, K. Robeyns, B. Norberg, J. Wouters and T. Leyssens, Cryst. Growth Des., 2014, 14, 3996-4004.

7 C. R. Groom, I. J. Bruno, M. P. Lightfoot and S. C. Ward, Acta Crystallogr., Sect. B: Struct. Sci., Cryst. Eng. Mater., 2016, 72, 171-179.

8 J. Dunitz, G. Filippini and A. Gavezzotti, Helv. Chim. Acta, 2000, 83, 2317-2335.

9 J. P. M. Lommerse, W. D. S. Motherwell, H. L. Ammon, J. D. Dunitz, A. Gavezzotti, D. W. M. Hofmann, F. J. J. Leusen, W. T. M. Mooij, S. L. Price, B. Schweizer, M. U. Schmidt, B. P. van Eijck, P. Verwer and D. E. Williams, Acta Crystallogr., Sect. B: Struct. Sci., 2000, 56, 697-714.

10 G. M. Day, W. D. S. Motherwell and W. Jones, Phys. Chem. Chem. Phys., 2007, 9, 1693-1704.

11 Cambridge Structural Database, CSD Space Group Statistics Space Group Frequency Ordering, 2017.

12 A. Gavezzotti, V. Colombo and L. Lo Presti, Cryst. Growth Des., 2016, 16, 6095-6104.

13 L. A. Nguyen, H. He and C. Pham-Huy, Int. J. Biomed. Sci., 2006, 2, 85.

14 Development of New Stereoisomeric Drugs, 1992, https://www. fda.gov/regulatory-information/search-fda-guidance-documents/ development-new-stereoisomeric-drugs.

15 T. Ward, Anal. Chem., 2002, 74, 2863-2872.

16 L. Pasteur, C. R. Hebd. Seances Acad. Sci., 1853, 162-166.

17 L. Pasteur, Ann. Chim. Phys., 1848, XXIV, 442-459.

18 E. Elacqua, Supramolecular chemistry of molecular concepts: tautomers, chirality, protecting groups, trisubstituted olefins, cyclophanes, and their impact on the organic solid state, $P h D$ thesis, University of Iowa, 2012.

19 P. Vishweshwar, J. McMahon, J. Bis and M. J. Zaworotko, J. Pharm. Sci., 2006, 95, 499-516.
20 E. Grothe, H. Meekes, E. Vlieg, J. H. ter Horst and R. de Gelder, Cryst. Growth Des., 2016, 16, 3043-3554.

21 C. Görbitz and H. Hersleth, Acta Crystallogr., Sect. B: Struct. Sci., 2000, 56(Pt 3), 526-534.

22 G. M. Crippen, Curr. Comput.-Aided Drug Des., 2008, 4, 259-264.

23 R. A. Valiulin, T. M. Arisco and A. G. Kutateladze, J. Org. Chem., 2012, 78, 2012-2025.

24 G. Springuel, B. Norberg, K. Robeyns, J. Wouters and T. Leyssens, Cryst. Growth Des., 2012, 12, 475-484.

25 T. Olszewska, A. Pyszno, M. J. Milewska, M. Gdaniec and T. Połoński, Tetrahedron: Asymmetry, 2005, 16, 3711-3717.

26 E. Grothe, H. Meekes and R. de Gelder, Acta Crystallogr., Sect. B: Struct. Sci., Cryst. Eng. Mater., 2017, 73, 453-465.

27 A. Cruz-Cabeza, S. Reutzel-Edens and J. Bernstein, Chem. Soc. Rev., 2015, 44, 8619-8635.

28 D. M. Zimmerman, B. E. Cantrell, J. K. Swartzendruber, N. D. Jones, L. G. Mendelsohn, J. D. Leander and R. C. Nickander, J. Med. Chem., 1988, 31, 555-560.

29 G. L. Perlovich, A. M. Ryzhakov, V. V. Tkachev, L. K. Hansen and O. A. Raevsky, Cryst. Growth Des., 2013, 13, 4002-4016.

30 T. Sun, CSD Communication (Private Communication), 2015.

31 S. Kiviniemi, M. Nissinen, T. Alaviuhkola, K. Rissanen and J. Pursiainen, J. Chem. Soc., Perkin Trans. 2, 2001, 2364-2369.

32 A. Rae and A. Willis, Z. Kristallogr. - Cryst. Mater., 2003, 218, 221-230.

33 B. Narymbetov, S. Khasanov, L. Zorina, L. Rozenberg, R. Shibaeva, D. Konarev and R. Lyubovskaya, Kristallografiya, 1997, 42, 851.

34 K. Kodama, H. Shitara and T. Hirose, Cryst. Growth Des., 2014, 14, 3549-3556.

35 L. Giri and V. Pedireddi, J. Mol. Struct., 2015, 1100, 455-463.

36 P. Briozzo, T. Kondo, K. Sada, M. Miyata and K. Miki, Acta Crystallogr., Sect. B: Struct. Sci., 1996, 52, 728-733.

37 L. Fábián and C. Brock, Acta Crystallogr., Sect. B: Struct. Sci., 2010, 66, 94-103.

38 C. C. da Silva and F. T. Martins, RSC Adv., 2015, 5, 20486-20490.

39 S. P. Kelley, L. Fábián and C. P. Brock, Acta Crystallogr., Sect. B: Struct. Sci., 2011, 67, 79-93.

40 T.-C. Chou, S.-Y. Chen and C. Yie-Hsung, Tetrahedron, 2003, 59, 9939-9950. 